\title{
Preoperational chronic pain impairs the attention ability before surgery and recovery of attention and memory abilities after surgery in non-elderly patients
}

This article was published in the following Dove Press journal:

Journal of Pain Research

\section{Huahua Gu',* \\ Xianyu Deng',* \\ Yizheng Lv' \\ Qian Chen' \\ Weifeng $\mathrm{Yu}^{3}$}

'Department of Anesthesiology, Huashan Hospital, Fudan University, Shanghai, China; ${ }^{2}$ Department of Neurosurgery, Tenth People's Hospital of Tongji University, Shanghai, China; ${ }^{3}$ Department of Anesthesiology, Renji Hospital, Shanghai Jiao Tong University School of Medicine, Shanghai, China

*These authors contributed equally to this work
Correspondence: Weifeng Yu Department of Anesthesiology, Renji Hospital, Shanghai Jiao Tong University School of Medicine, 160 Pujian Road, Shanghai 200127, China

Tel +86 I39 01961704

Email ywf808@yeah.net
Purpose: This study aimed to investigate the relationship of preoperative chronic pain and postoperative cognitive dysfunction (POCD) in non-elderly patients who underwent arthroscopic surgery. Materials and methods: The pain intensity was estimated using visual analog scale, and the cognitive function was assessed by Syndrom Kurz Test. The effects of preoperative chronic pain on the cognitive scales were comparatively studied between the patients of observational group (OG, with chronic pain) and control group (CG, without chronic pain) pre- and postoperatively, and followed up for 3 months.

Results: A total of 57 non-elderly patients completed the study. Twenty-five patients (44\%) with preoperative chronic pain were assigned to OG and 32 patients $(56 \%)$ without chronic pain were assigned to CG. Preoperation chronic pain impaired the attention ability before surgery and caused less recovery of attention and memory abilities from 24 hours to 3 months after the surgery. Surgery procedures improved the attention and memory abilities and impaired the ability of numerical ability in CG patients. A postoperative pain relief in OG patients caused more recovery of cognition in addition to surgery procedure-mediated cognitive recovery. The incidence of POCD was $\sim 3.5 \%$ and temporary at 24 hours after surgery, and disappeared at 2 weeks, 6 weeks and 3 months after the surgery.

Conclusion: The incidence of POCD in non-elderly population who underwent arthroscopic surgery was low. Surgery improved the abilities of attention and memory, and impaired the ability of counting. The preoperative chronic pain distracted the attention before surgery, and reduced the recovery of attention and memory abilities during the follow-up period after the surgery in non-elderly patients.

Keywords: cognition dysfunction, chronic pain, non-elderly population

\section{Introduction}

Postoperative cognitive dysfunction (POCD) is recently well recognized as a common clinical phenomenon after anesthesia and surgery, ${ }^{1,2}$ which is characterized by a temporary decline in cognitive function in weeks or months following a surgical procedure. ${ }^{3,4}$ Age is a major risk factor for POCD because it occurs more frequently in the elderly population., ${ }^{5,6}$ The incidence and severity of POCD in the younger population is not well studied. It is necessary to answer whether the anesthesia and surgery will influence the work and live ability of young adults.

In addition to age, several risk factors have been investigated and related to POCD, such as low educational level, pre-existing cognitive impairment, major surgery and type 
of anesthesia. ${ }^{2,7}$ Previous clinical studies have demonstrated that postoperative pain and analgesic techniques could influence postoperative cognitive status. ${ }^{8}$ In animal study, postoperative pain can impair the performance of aging rats with working and reference memory tasks. ${ }^{4}$ Ozgür et al indicated that pre-existing pain prior to surgery has an influence on the postoperative pain course. ${ }^{9}$ Chronic pain is defined as pain that lasts longer than 6 weeks after the primary injury may have disappeared or significantly subsided. Patients with chronic pain have a poor performance on attentional and mnemonic tasks. ${ }^{10,11}$ However, it is not clear whether preoperative chronic pain has an effect on POCD in non-elderly population.

This study aimed to investigate the relationship of preoperative chronic pain and POCD in non-elderly population who underwent arthroscopic surgery.

\section{Materials and methods}

\section{Patients}

This research is a prospective cohort study of POCD in nonelderly patients with or without chronic pain. Collection and review of patient data in the present study were approved by the Huashan Committee on Human Research at Fudan University, and informed consent was obtained from each subject. Patients with drug or alcohol abuse, history of head injury, attention deficits, and neurological or psychological disorder were excluded. Eligible individuals were 18-59 years old and well-educated (primary school or higher). Initially, 73 patients who were admitted in Huashan Hospital for arthroscopic surgery during January to December 2016 were included in the study. Sixteen patients $(21.9 \%)$ did not complete the assessments and 57 patients completed the study. On the basis of the visual analog scale (VAS), 25 patients with chronic pain were assigned to the observation group (OG) and 32 patients without chronic pain were assigned to the control group (CG).

\section{Visual analog scale}

The VAS anchored at 0 and 10 was used to measure pain intensity. ${ }^{11}$ A score of 0 in the VAS was designated as grade 1 , scores of 1-3 were designated as grade 2, scores of 4-7 were designated as grade 3 and scores of 8-10 were designated as grade 4 . Patients with a baseline pain of at least 4 on a VAS (grade 3 or 4) and lasting for more than 6 weeks were assigned into the $\mathrm{OG}$, and the patients without chronic pain (grade 1 or 2 ) were assigned to the $\mathrm{CG}$.

\section{Arthroscopic surgery}

Arthroscopic surgery was performed according to the standard procedures including arthroscopic clean-up, rotator cuff repair, acromioplasty and cheiloplasty. All patients were treated without differences in anesthesia time, operation time, and the amount of fentanyl, remifentanil, ropivacaine and betamethasone used during the arthroscopic surgery between OG and CG (Table 1).

\section{Syndrom Kurz Test}

Cognitive function was assessed with the use of Syndrom Kurz Test (SKT), a short cognitive performance test for evaluating deficits of memory and attention. ${ }^{12,13}$ The SKT consists of nine subtests, including naming objects (I), immediate recall (II), naming numerals (III), arranging blocks (IV), replacing blocks (V), counting symbols (VI), reversal naming (VII), delayed recall (VIII) and recognition memory (IX). All time consumption was measured by a stopwatch and the patients were asked to work as fast and accurately as possible. Each subtest was limited to 60 seconds and the total administration time rarely exceeds 15 minutes. The test was carried out in a quiet room. Patients were assessed by SKT on the day before surgery, 24 hours after surgery, 2 weeks after surgery, 6 weeks after surgery and 3 months after surgery. Five parallel versions of SKT were given in random order throughout follow-up.

All group data were used to calculate mean and SD of each subtest. The individual result of each subtest was subtracted by the corresponding mean, and divided by SD to obtain a standardized score. If the average of the followup standardized scores of nine subtests was increased by 1

Table I Treatment during the joint arthroscopic surgery

\begin{tabular}{|l|l|l|l|}
\hline Intraoperative situation & OG $(\mathbf{n = 2 5})$ & CG $(\mathbf{n}=\mathbf{3 2})$ & $\boldsymbol{P}$-value \\
\hline Anesthesia time (minutes) (mean \pm SD) & $96.0 \pm 39.6$ & $110.4 \pm 53.0$ & 0.264 \\
\hline Operation time(minutes) (mean \pm SD) & $66.5 \pm 33.2$ & $78.3 \pm 44.8$ & 0.279 \\
\hline Fentanyl $(\mu \mathrm{g})($ mean \pm SD) & $386.8 \pm 79.5$ & $435.6 \pm 89.3$ & $0.764^{*}$ \\
\hline Remifentanil $(\mu \mathrm{g})$ (mean \pm SD) & $433.6 \pm 240.1$ & $571.3 \pm 495.1$ & $0.728^{*}$ \\
\hline Ropivacaine + betamethasone (treated vs untreated) & 19.6 & $20: 12$ & 0.391 \\
\hline
\end{tabular}


compared with the preoperative average standardized score, the patient was diagnosed as POCD.

\section{Statistical analysis}

Statistical analysis was performed with SPSS 22.0 (IBM Corporation, New York, NY; formerly SPSS Inc., Chicago, IL). Continuous data were presented as mean \pm SD or median (IQR).

The significant differences between groups were tested using the Student's $t$-test if the data were normally distributed, and with Wilcoxon two sample test if the data were not normally distributed. Repeated-measures ANOVA was performed to determine the significance of the differences of the postoperational SKT scores between the groups of the patients. The comparison of preoperational SKT scores with the corresponding postoperational SKT scores was tested using the paired sample $t$-test or Wilcoxon signed-rank test. Chi-squared test or Fisher's exact test was performed to determine the significance of the difference between two groups of discrete data. All tests were two-tailed, and $P<0.05$ was taken as statistically significant.

\section{Ethics approval}

Ethical approval was given by the Huashan Committee on Human Research at Fudan University.

\section{Results}

\section{Demographic characteristics of the patients}

There was no difference in gender composition between OG and CG. The patients of OG were significantly $(P<0.05)$ older than those of CG. The body weight and height of the patients of $\mathrm{OG}$ were significantly $(P<0.05)$ lower than those of CG. There was significant $(P<0.05)$ difference in the type of organs surgically treated between OG and CG. There was no difference in the duration of anesthesia and surgery, education, arterial pressure, heart rate, respiration rate or body temperature between OG and CG (Table 2).

\section{SKT subtest scores of OG and CG before and after surgery}

There were significantly $(P<0.05)$ higher scores of SKT I, V, VI and VII in OG than CG before surgery (Table 3). There was no difference in the scores of SKT II, III, IV, VIII or IX between OG and CG before surgery (Table 3). Twentyfour hours after the surgery, scores of SKT VI and VII were significantly $(P<0.05)$ higher in OG than CG (Table 3$)$. Two weeks after the surgery, scores of SKT V and VII were significantly $(P<0.05)$ higher in OG than CG (Table 3$)$. Six weeks after the surgery, scores of SKT I, V, VI and VII were significantly $(P<0.05)$ higher in OG than CG (Table 3$)$. Three months after the surgery, scores of SKT V and VII were significantly $(P<0.05)$ higher in OG than $\mathrm{CG}$ (Table 3$)$. There were significant $(P<0.05)$ decreases in the scores of SKT III 24 hours after the surgery, SKT I, III, IV, VII and VIII 2 weeks after the surgery, SKT I, II, III and IV 6 weeks after the surgery, and SKT I, II, III, IV, V, VII and VIII 3 months after the surgery, and significant $(P<0.05)$ increases in the scores of SKT VI and VIII 24 hours after the surgery, and SKT VI 6 weeks after the surgery, when compared with the corresponding presurgery SKT scores, in the OG patients

Table 2 Demographic and clinical characteristics of the patients

\begin{tabular}{|c|c|c|c|}
\hline Parameters & OG $(n=25)$ & CG $(n=32)$ & $P$-value \\
\hline Sex, n (\%) & & & 0.213 \\
\hline Male & $14(56.00)$ & $23(28.13)$ & \\
\hline Female & II (44.00) & $9(7 \mid .88)$ & \\
\hline Age (years), mean \pm SD & $39.9 \pm 9.9$ & $33.6 \pm 8.7$ & $0.006 * *$ \\
\hline Height $(\mathrm{cm})$, mean \pm SD & $166.6 \pm 10.0$ & $172.7 \pm 9.0$ & $0.019 *$ \\
\hline Body weight $(\mathrm{kg})$, mean $\pm S D$ & $65.2 \pm 12.9$ & $75.3 \pm 16.1$ & $0.013^{*}$ \\
\hline Education, n (\%) & & & 0.257 \\
\hline Secondary school and below & $7(28.0)$ & $4(12.5)$ & \\
\hline University and above & $18(72.0)$ & $28(87.5)$ & \\
\hline Surgery sites, n (\%) & & & $<0.001 * *$ \\
\hline Shoulder & $8(32.00)$ & $I(3.13)$ & \\
\hline Knee & $10(40.00)$ & $29(90.63)$ & \\
\hline Ankle & $7(28.00)$ & $2(6.25)$ & \\
\hline Arterial pressure $(\mathrm{mmHg})$, mean $\pm \mathrm{SD}$ & $95.5 \pm 9.4$ & $92.2 \pm 12.3$ & 0.452 \\
\hline Heart rate $(\mathrm{bpm})$, mean \pm SD & $75.4 \pm 9.8$ & $74.3 \pm 10.1$ & 0.686 \\
\hline Respiration (bpm), mean \pm SD & $17.0 \pm 1.6$ & $16.7 \pm 1.5$ & 0.463 \\
\hline Body temperature $\left({ }^{\circ} \mathrm{C}\right)$, mean $\pm S D$ & $36.7 \pm 0.3$ & $36.6 \pm 0.3$ & 0.424 \\
\hline
\end{tabular}

Notes: *Comparison of the parameters between $O G$ and $C G, P<0.05$; **comparison of the parameters between $O G$ and $C G, P<0.0$ I.

Abbreviations: CG, control group; OG, observational group. 


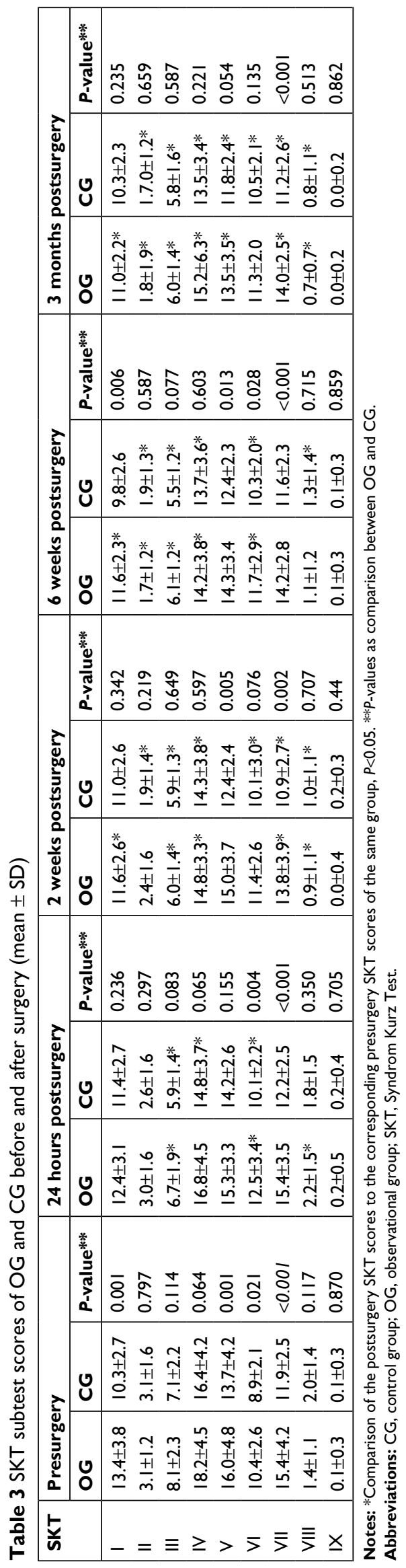

(Table 3). There were significant $(P<0.05)$ decreases in the scores of SKT III and IV 24 hours after the surgery, SKT II, III, IV, VII and VIII 2 weeks after the surgery, SKT II, III, IV and VIII 6 weeks after the surgery, and SKT II, III, IV, V, VII and VIII 3 months after the surgery, and significant $(P<0.05)$ increases in the scores of SKT VI 24 hours after the surgery, SKT VI 2 weeks after the surgery, SKT VI 6 weeks after the surgery, and SKT VI 3 months after the surgery, when compared with the corresponding presurgery SKT scores, in the CG patients (Table 3$)$. There were significantly $(P<0.05)$ more decreases in the scores of SKT I at 24 hours, 2 weeks and 3 months after surgery in OG than CG when compared with the corresponding presurgery SKI scores (Table 4).

\section{VAS scores of OG and CG before and after surgery}

All OG patients had VAS III or VAS IV pain while all CG patients had a pain less than VAS II before surgery (Table 5). The VAS scores significantly $(P<0.05)$ decreased at 24 hours, 2 weeks, 6 weeks and 3 months after surgery when compared with the VAS score before surgery in OG (Figure 1). The preoperation VAS score was significantly $(P<0.05)$ higher in OG than CG (Figure 1). All OG patients had VAS III or VAS IV pain while all CG patients had a pain less than VAS II before surgery. There was no difference among the VAS scores before and after surgery in CG (Figure 1). There was no difference among the VAS scores after surgery between OG and CG (Figure 1, Table 5).

\section{Incidence of POCD in OG and CG after surgery}

The incidence of POCD with preoperative chronic pain was $4 \%(1 / 25)$ in OG which is similar to CG $(3 \%, 1 / 32)$. Cognitive dysfunction of these two patients was recovered in 2 weeks after surgery. The incidence of POCD with or without chronic pain at 2 weeks after surgery, 6 weeks after surgery and 3 months after surgery were all zeros.

\section{Discussion}

This study for the first time investigated whether chronic pain influenced the incidence and severity of POCD in non-elderly population. It was found that the patients with preoperation chronic pain had poorer cognition than the patients without preoperation chronic pain before and after the surgery. Both the patients with and without preoperation chronic pain decreased cognition 24 hours after the surgery, increased the cognition 2 weeks, 6 weeks and 3 months after 


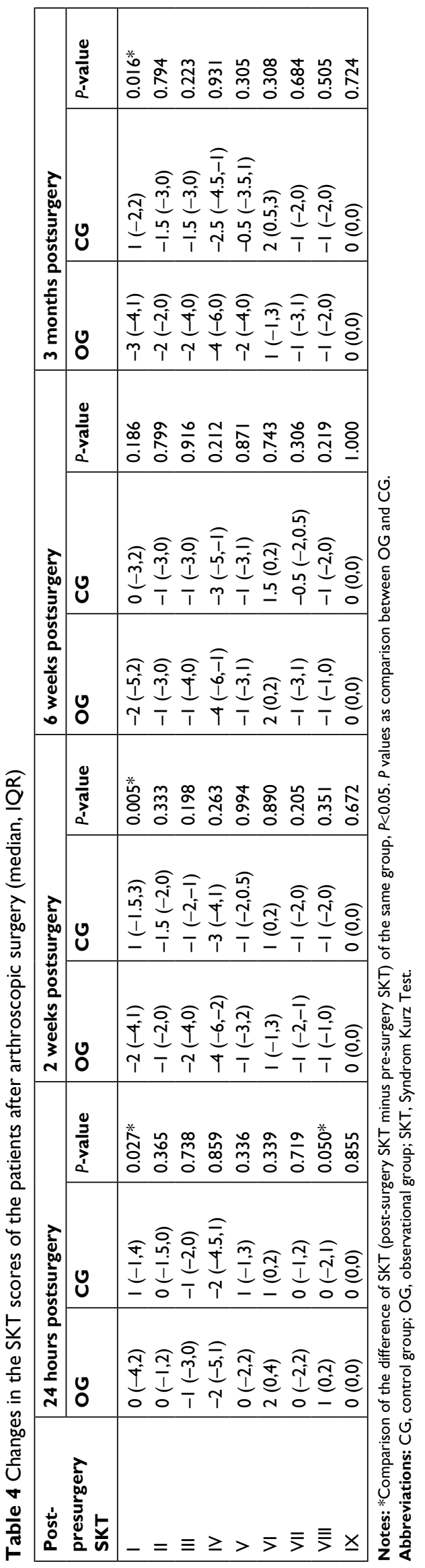

Table 5 VAS scores of the patients after arthroscopic surgery

\begin{tabular}{|c|c|c|c|c|}
\hline Time & VAS & $\begin{array}{l}\text { OG } \\
(n=25)\end{array}$ & $\begin{array}{l}\text { CG } \\
(n=32)\end{array}$ & $P$-value \\
\hline \multirow[t]{2}{*}{ Presurgery } & $0-2$ & I (4.00) & $32(100.0)$ & 0.000 \\
\hline & $3-4$ & $24(96.00)$ & $0(0.00)$ & \\
\hline \multirow[t]{2}{*}{24 hours postsurgery } & $0-2$ & $24(96.00)$ & $27(84.38)$ & 0.215 \\
\hline & $3-4$ & I (4.00) & $5(15.63)$ & \\
\hline \multirow[t]{2}{*}{2 weeks postsurgery } & $0-2$ & $23(92.00)$ & $32(100.0)$ & 0.188 \\
\hline & $3-4$ & $2(8.00)$ & $0(0.00)$ & \\
\hline \multirow[t]{2}{*}{6 weeks postsurgery } & $0-2$ & $23(92.00)$ & $32(100.0)$ & 0.188 \\
\hline & $3-4$ & $2(8.00)$ & $0(0.00)$ & \\
\hline \multirow[t]{2}{*}{3 months postsurgery } & $0-2$ & $25(100.0)$ & $32(100.0)$ & 1.000 \\
\hline & $3-4$ & $0(0.0)$ & $0(0.0)$ & \\
\hline
\end{tabular}

Abbreviations: CG, control group; OG, observational group; VAS, visual analog scale.

the surgery. The patients with preoperation chronic pain had more improvement on naming objects at 24 hours, 2 weeks and 3 months after surgery than the patients without chronic pain. No difference in the incidence of POCD was found between patients with preoperation chronic pain and the patients without preoperation chronic pain. Preoperation chronic pain impaired the cognition before surgery and reduced recovery of cognition after surgery in non-elderly patients with arthroscopic surgery.

\section{Pain of the patients}

The VAS scores indicated that all OG patients had a grade 3 or 4 preoperational chronic pain while CG patients had a non-chronic pain lower than grade 2 . The pain of the OG patients decreased to the same level as the CG patients at 24 hours, 2 weeks, 6 weeks and 3 months after the surgery. The pain of the CG patients did not change before and after the surgery.

\section{Cognition of the patients}

Higher scores of SKT I, V, VI and VII in OG than CG indicated that $\mathrm{OG}$ patients had poorer abilities in naming objects, replacing blocks, counting symbols and reversal naming than CG patients before surgery, suggesting that OG patients had less attention ability than CG patients before surgery. Higher scores of SKT VI and VII at 24 hours after the surgery, SKT V and VII at 2 weeks after the surgery, SKT I, V, VI and VII at 6 weeks after the surgery and SKT V and VII at 3 months after the surgery in OG than CG implicated that OG patients had poorer abilities in counting symbols and reversal naming 24 hours after the surgery, replacing blocks and reversal naming 2 weeks after the surgery, naming objects, replacing blocks, counting symbols and reversal naming 6 weeks after the surgery, and replacing blocks and reversal naming 3 months after the surgery than CG patients. These results 


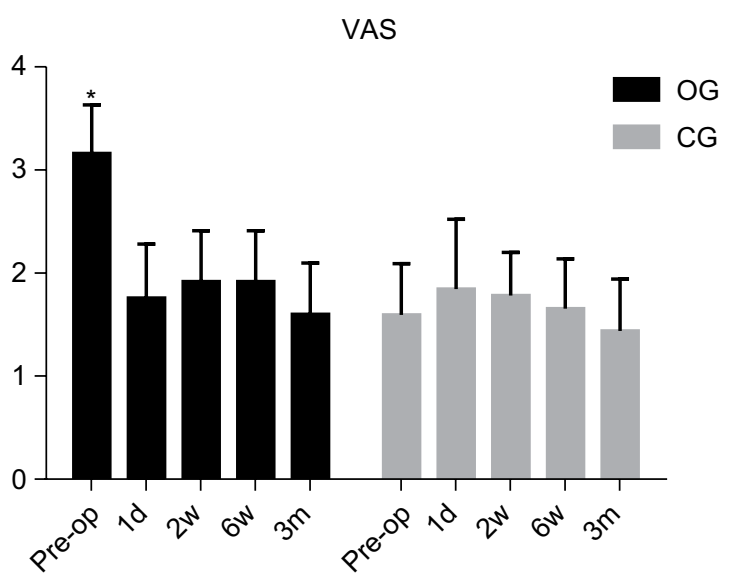

Figure I Comparison of the pain scores between the groups at preoperation, 24 hours, 2 weeks, 6 weeks and 3 months after operation.

Notes: *Comparison of the OG preoperation VAS score with the CG preoperation VAS score, and comparison of the preoperation VAS score with the VAS scores at 24 hours, 2 weeks, 6 weeks and 3 months after operation in the OG $(P<0.05)$.

Abbreviations: CG, control group; OG, observational group; VAS, visual analog scale.

suggest that OG patients should have less attention ability than CG patients within 3 months after surgery.

As compared to the corresponding presurgery cognitive abilities, a decrease in the score of SKT III and increases in the scores of SKT VI and VIII indicated that the OG patients improved the ability in naming numerals and decreased the abilities in counting symbols and recalling at 24 hours after the surgery; a decrease in the scores of SKT I, III, IV, VII and VIII 2 weeks after the surgery implicated that OG patients improved the abilities in naming objects, naming numerals, arranging blocks, reversal naming and delayed recall 2 weeks after the surgery; a decrease in the scores of SKT I, II, III and IV and an increase of the score of SKT VI indicated that OG patients improved the abilities in naming objects, immediate recall, naming numerals and arranging blocks, and decreased the ability in counting symbols, 6 weeks after the surgery; and a decrease in the scores of SKT I, II, III, IV, V, VII and VIII implicated that the OG patients improved the abilities in naming objects, immediate recall, naming numerals, arranging blocks, replacing blocks, reversal naming and delayed recall 3 months after the surgery, when compared with the presurgery status of cognition. These observations suggest that OG patients decreased abilities of memory and counting at 24 hours after the surgery, increased abilities of attention and memory from 2 weeks to three months after the surgery, and decreased the ability of counting 6 weeks after the surgery.

As compared to the corresponding presurgery SKT scores, a decrease in the scores of SKT III and IV and an increase in the scores of SKT VI implicated that the CG patients improved the abilities in naming numerals and arranging blocks and decreased the ability in counting symbols, 24 hours after the surgery; a decrease in the scores of SKT II, III, IV, VII and VIII, and an increase in the score of SKT VI implicated that the CG patients improved the abilities in immediate recall, naming numerals, arranging blocks, reversal naming and delayed recall, and decreased the ability in counting symbols, 2 weeks after the surgery; a decrease in the scores of SKT II, III, IV and VIII, and an increase in the score of SKT VI, suggest that the CG patients improved the abilities in immediate recall, naming numerals, arranging blocks and delayed recall, and decreased the ability in counting symbols, 6 weeks after the surgery; a decrease in the scores of SKT II, III, IV, V, VII and VIII, and an increase in the score of SKT VI 3 months after the surgery, implicated that the $\mathrm{CG}$ patients improved the abilities in immediate recall, naming numerals, arranging blocks, replacing blocks, reversal naming and delayed recall, and decreased the ability in counting symbols, 3 months after the surgery. These results suggest that CG patients improved the attention ability 24 hours after the surgery, the abilities of attention and memory 2 weeks, 6 weeks and 3 months after the surgery, but decreased ability of counting after surgery.

A more decrease in the scores of SKT I at 24 hours, 2 weeks and 3 months after surgery in OG than CG when compared with the corresponding presurgery SKI scores implicated that OG patients improved more ability in naming objects than $\mathrm{CG}$ patients, suggesting that $\mathrm{OG}$ patients improved more attention ability than $\mathrm{CG}$ patients after the surgery.

\section{Effects of pain on cognition}

The average incidence of POCD was $\sim 3.5 \%$ in non-elderly patients with arthroscopic surgery. No difference in the incidence of POCD was found between patients with preoperation chronic pain and the patients without preoperation chronic pain, suggesting that preoperational chronic pain did not increase the incidence of POCD.

Patients from OG had a poorer cognition than CG preoperatively, especially the poorer attention, implicating that preoperational pain impaired the cognition of the patients before the operation. Patients from OG had a poorer cognition than CG after the operation while both OG and CG patients had the same level of pain, uncovered that preoperational chronic pain impaired the recovery of cognition until 3 months after the operation. Since the pain of the CG patients did not change before and after the surgery, the changes in the SKT scores after the operation reveals that operation procedures 
improved the cognition abilities in naming numerals and arranging blocks at 24 hours after the surgery, the abilities in immediate recall, naming numerals, arranging blocks, reversal naming and delayed recall 2 weeks after operation, the abilities in immediate recall, naming numerals, arranging blocks and delayed recall 6 weeks after the operation, and the abilities in immediate recall, naming numerals, arranging blocks, replacing blocks, reversal naming and delayed recall 3 months after the operation, and impaired the ability in counting symbols at 24 hours, 2 weeks, 6 weeks and 3 months after the operation. These results suggest that surgery improved the attention ability at 24 hours after the surgery, the abilities of attention and memory 2 weeks, 6 weeks and 3 months after the surgery, and impaired the ability of counting within 3 months after the surgery. The presence of a decrease in the scores of SKT I only in OG patients but not in the CG patients at 2 weeks, 6 weeks and 3 months after the operation and a more decrease in the score of SKT I at 24 hours, 2 weeks and 3 months after surgery in OG than CG when compared with the corresponding presurgery SKI scores, uncovered that operation-mediated pain relief improved the ability in naming objects, which means increased ability of attention. The presence of the increase in the scores of SKT IV at 24 hours and 6 weeks after the operation but not at 2 weeks or 3 months after the operation suggests that the operation procedure-mediated impairment in attention ability was compromised in the OG patients at 2 weeks and 3 months after the operation. The presence of the increase in the score of SKT VIII and the absence of the decrease in the score of SKT IV in OG patient but not CG patients at 24 hours after the operation indicated that preoperation chronic pain impaired the ability of delayed memory and the improvement of the ability in attention at 24 hours after the operation. The presence of a decrease in the score of SKT VIII in the CG patients but not the OG patients at 6 weeks after the operation revealed that preoperational pain compromised the operation procedure-mediated recovery of the ability of memory.

\section{Risk factors for POCD}

POCD is a common problem of modern medicine, which causes delay of discharge and rehabilitation. Risk factors for POCD include advanced age, low educational level, preexisting cognitive impairment, major surgery and general anesthesia. Age is the most substantial risk factor. ${ }^{14,15} \mathrm{~A}$ larger number of studies have indicated that the POCD occurs frequently in elder people. ${ }^{14,15}$ Although there were differences in the age and surgery sites between the OG and CG patients, no difference in the incidence of POCD between the two groups indicates that the age and surgery site differences between these two groups did not cause POCD in the non-elderly patients. The incidence of cognitive dysfunction in this study was $3.5 \%$, which was significantly lower than the $19.2 \%$ observed in the population aged $40-59$ years $^{6}$ and $25.8 \%$ observed in the population aged over 60 years. ${ }^{5}$ These differences may be because that the average age of the target patients in this study was 36 years old and dramatically younger than the patients in the previous study. ${ }^{16}$ Another reason may be that the cardiac surgery ${ }^{17}$ and major non-cardiac surgery ${ }^{5}$ in the previous studies were relatively more invasive than the arthroscopic surgery used in this study. Since there was no difference in the anesthesia treatment between the groups of patients, it is still unclear whether generalized anesthesia influences the ability of memory and attention in non-elderly population. Perioperative pain is closely linked to postoperative cognitive impairment, and is also an essential risk factor for cognitive dysfunction. ${ }^{18,19}$

Chi et al documented that postoperative pain is one of the risk factors for POCD, ${ }^{4}$ which is hard to be verified in this study as there was no difference in the pain levels between OG and CG patients after the surgery. Ozgür et al indicated that pre-existing pain prior to surgery has an influence on the postoperative pain course. ${ }^{9}$ No difference in the incidence of POCD between OG and CG indicated that preoperational chronic pain did not influence the occurrence of POCD in non-elderly patients. It was observed that the cognition level of the OG patients was lower than that in the CG patients both preoperatively and postoperatively, which is in accordance with the observations by Berryman et al and Tarasidis et al, ${ }^{10,20,21}$ suggesting that preoperational chronic pain may damage the top-down control ability, distract people's attention ${ }^{22}$ and impair the recovery of cognition after surgery. ${ }^{9}$

It should be cautiously mentioned here that the not wellmatched age and surgery site, which are risk factors for POCD,${ }^{14,15,17}$ may influence the interpretation of the results. The study may also be limited by the small sample size. A considerable sample size may be required to be enough to detect the difference in the incidence of POCD between the patients with and without chronic pain.

\section{Conclusion}

The incidence of POCD in non-elderly population who underwent arthroscopic surgery was low. Surgery improved the abilities of attention and memory and impaired the ability of counting. The preoperative chronic pain did not further deteriorate postoperative cognitive impairment, but it did distract the attention before the surgery and reduced the recovery of memory and attention abilities after the operation. 


\section{Consent for publication}

All patients gave their written informed consent.

\section{Data sharing statement}

The datasets used and/or analyzed during the current study are available from the corresponding author on reasonable request.

\section{Acknowledgments}

This work was supported by National Natural Science Foundation of China, grant number 81571048 .

\section{Disclosure}

The authors report no conflicts of interest in this work.

\section{References}

1. Newman S, Stygall J, Hirani S, Shaefi S, Maze M. Postoperative cognitive dysfunction after noncardiac surgery: a systematic review. Anesthesiology. 2007;106:1228-1234.

2. Rundshagen I. Postoperative cognitive dysfunction. Dtsch Arztebl Int. 2014;111(8):119-125.

3. Pandharipande PP, Girard TD, Jackson JC, et al. Long-term cognitive impairment after critical illness. New Engl J Med. 2014;370:1306-1316.

4. Chi H, Kawano T, Tamura T, et al. Postoperative pain impairs subsequent performance on a spatial memory task via effects on N-methyl-Daspartate receptor in aged rats. Life Sci. 2013;93(25-26):986-993.

5. Moller JT, Cluitmans P, Rasmussen LS, et al. Long-term postoperative cognitive dysfunction in the elderly ISPOCD1 study. ISPOCD investigators. International Study of Post-Operative Cognitive Dysfunction. Lancet. 1998;351(9106):857-861.

6. Johnson T, Monk T, Rasmussen LS, et al. Postoperative cognitive dysfunction in middle-aged patients. Anesthesiology. 2002;96(6):1351-1357.

7. Feinkohl I, Winterer G, Spies CD, Pischon T. Cognitive reserve and the risk of postoperative cognitive dysfunction. Dtsch Arztebl Int. 2017;114(7):110.
8. Fong HK, Sands LP, Leung JM. The role of postoperative analgesia in delirium and cognitive decline in elderly patients: a systematic review. Anesth Analg. 2006;102(4):1255-1266.

9. Özgür E, Straub K, Wille S, Engelmann U, Dagtekin O, Gerbershagen HJ. Impact of preoperative pain on postoperative pain chronification. Urologe A. 2011;50:1396-1402.

10. Dick BD, Rashiq S. Disruption of attention and working memory traces in individuals with chronic pain. Anesth Analg. 2007;104(5):1223-1229.

11. Li L, Liu X, Herr K, Llm RN. Postoperative pain intensity assessment: a comparison of four scales in Chinese adults. Pain Med. 2007;8(3):223-234.

12. Overall JE, Schaltenbrand R. The SKT neuropsychological test battery. J Geriatr Psychiatry Neurol. 1992;5(4):220-227.

13. Lehfeld H, Erzigkeit H. The SKT - a short cognitive performance test for assessing deficits of memory and attention. Int Psychogeriatr. 1997;9(Suppl 1):115-121.

14. Kotekar N, Kuruvilla CS, Murthy V. Post-operative cognitive dysfunction in the elderly: a prospective clinical study. Indian J Anaesth. 2014;58(3):263-268.

15. Xu T, Bo L, Wang J, et al. Risk factors for early postoperative cognitive dysfunction after non-coronary bypass surgery in Chinese population. J Cardiothorac Surg. 2013;8(1):1-6.

16. Wang $\mathrm{W}$, Wang $\mathrm{Y}, \mathrm{Wu} \mathrm{H}$, et al. Postoperative cognitive dysfunction: current developments in mechanism and prevention. Int Med J Exper Clin Res. 2014;20:1908-1912.

17. Gao L, Taha R, Gauvin D, Othmen LB, WangY, Blaise G. Postoperative cognitive dysfunction after cardiac surgery. Chest. 2005;128(5):3664-3670.

18. Eü B, Kaya M, Şenel GÖ, Ünver S. The incidence of delirium at the postoperative intensive care unit in adult patients. Turk J Anaesthesiol Reanimation. 2015;43:232.

19. Lin YT, Lan KM, Wang LK, et al. Incidence, risk factors, and phenomenological characteristics of postoperative delirium in patients receiving intravenous patient-controlled analgesia: a prospective cohort study. Neuropsychiatr Dis Treat. 2016;12:3205-3212.

20. Berryman C, Stanton TR, Jane Bowering K, Tabor A, McFarlane A, Lorimer Moseley G. Evidence for working memory deficits in chronic pain: a systematic review and meta-analysis. Pain. 2013;154(8):1181-1196.

21. Tarasidis GS, Deconde AS, Mace JC, et al. Cognitive dysfunction associated with pain and quality of life in chronic rhinosinusitis. Int Forum Allergy Rhinol. 2015;5(11):1004-1009.

22. Millan MJ. Descending control of pain. Prog Neurobiol. 2002;66(6): 355-474.
Journal of Pain Research

\section{Publish your work in this journal}

The Journal of Pain Research is an international, peer reviewed, open access, online journal that welcomes laboratory and clinical findings in the fields of pain research and the prevention and management of pain. Original research, reviews, symposium reports, hypothesis formation and commentaries are all considered for publication.

\section{Dovepress}

The manuscript management system is completely online and includes a very quick and fair peer-review system, which is all easy to use. Visit http://www.dovepress.com/testimonials.php to read real quotes from published authors. 